\title{
Twin Zygosity Diagnosis by Genetic Systems-An Efficiency Evaluation A Comment to a Paper by Selvin [1977]
}

\section{Ranajit Chakraborty}

Center for Demographic and Population Genetics, University of Texas Health Science Center, Houston

The importance of genetic markers in the diagnosis of twin zygosity is well emphasized in genetic literature [see, eg, 3]. It is well known that when members of a twin pair are discordant for a genetic marker the pair is classified as dizygotic (DZ), while the pairs that are concordant for all systems studied are assumed to be monozygotic (MZ). Given this rule, it is obvious that the genetic systems to be studied for zygosity determination of a random twin pair are to be judiciously chosen to minimize the error of misclassification.

Recently, Selvin [3] proposed a measure of efficiency to evaluate the relative effectiveness of the different genetic markers. Through some numeric evaluations, he concluded that the more complex multiallelic systems are not necessarily more efficient as compared to a two-allele autosomal marker for determining zygosity of a random twin pair when the parental genotypes are not known. This conclusion appeared to be quite contradictory to simple intuitive arguments. The purpose of this note is to point out some of the pitfalls of Selvin's study.

Let us first note that the information regarding twin zygosity provided by a genetic system is first evaluated through the computation of the probability of achieving concordance given that the twin pair is DZ. Neel and Schull [1] and Smith and Penrose [4] discuss computations of this probability in great detail. For example, as Selvin noted, in a diallelic autosomal locus where all genotypes are phenotypically recognizable (as in most enzyme systems studied electrophoretically or in blood group systems like Kell and $\mathrm{Kidd}$ using antisera $-\mathrm{K}$ and $-\mathrm{k}$, and $-\mathrm{JK}^{\mathrm{a}}$ and $-\mathrm{Jk}^{\mathrm{b}}$ ) this probability is given by:

$$
\text { Prob(concordance } \mid \mathrm{DZ})=1-\mathrm{pq}(4-3 \mathrm{pq}) / 2
$$

where $p$ and $q(=1-p)$ are the two gene frequencies. Clearly, a minimum value of this probability is reached when $p=q=1 / 2$. 
On the contrary, when one of the alleles is dominant over the other, as may be the case for the blood groups $\mathbf{P}$ (tested only with $-\mathrm{P}$ antiserum), Lutheran (usually tested with $-\mathrm{Lu}^{\mathrm{a}}$ antiserum) and the like, the probability of concordance is given by:

$$
\text { Prob(concordance } \mid \mathrm{DZ})=1-\mathrm{pq}^{2}(3+\mathrm{q}) / 2
$$

As Smith and Penrose [4] indicated, the general procedure of evaluating such probabilities for any genetic system is to set out the joint phenotypic distribution of two sibs (which should be the same for members of a DZ twin pair) and add over the probabilities of all diagonal entries. The minimum value of Prob(concordance IDZ) for such a system is obtained for $q=0.686$ (differentiating the expression in Equation 2 with respect to $q$, treating $p$ as $1-q$, and setting the differential to zero to obtain a solution for $q$ ). The minimum value of the probability of concordance for a diallelic autosomal dominant locus, therefore, is 0.7277 and not 0.5938 (as would occur if we were dealing with the expression in Equation 1). To contrast the effect of dominance on Selvin's measure of efficiency, we plot the efficiency as a function of gene frequency for two-allele autosomal loci with and without dominance relationship between alleles in the Figure. It indicates that, so long as the gene frequency of the recessive allele is more than 0.63 , a diallelic autosomal recessive locus is more "efficient" than its codominant counterpart. On the other hand, the probability of concordance as given in Equation 1 is always less than that of Equation 2 (unless $p=0$ or 1) which indicates that, for a fixed set of gene frequencies, an autosomal codominant locus is always more informative than a diallelic autosomal recessive locus. This questions the appropriateness of Selvin's measure of efficiency.

Furthermore, for the gene frequencies considered by Smith and Penrose [4] at ABO, $\mathrm{A}_{1} \mathrm{~A}_{2} \mathrm{BO}, \mathrm{MNSs}$, and $\mathrm{Rh}$ loci, the probabilities of concordance were inadvertently miscalculated by Selvin. By summing the diagonal entries of the sib-sib joint phenotype frequencies of Smith and Penrose [4: Tables 2, 4, and 6] I obtain the correct Prob (concordance $\mid \mathrm{DZ}$ ) for these systems which are shown in the the Table below. The minimum

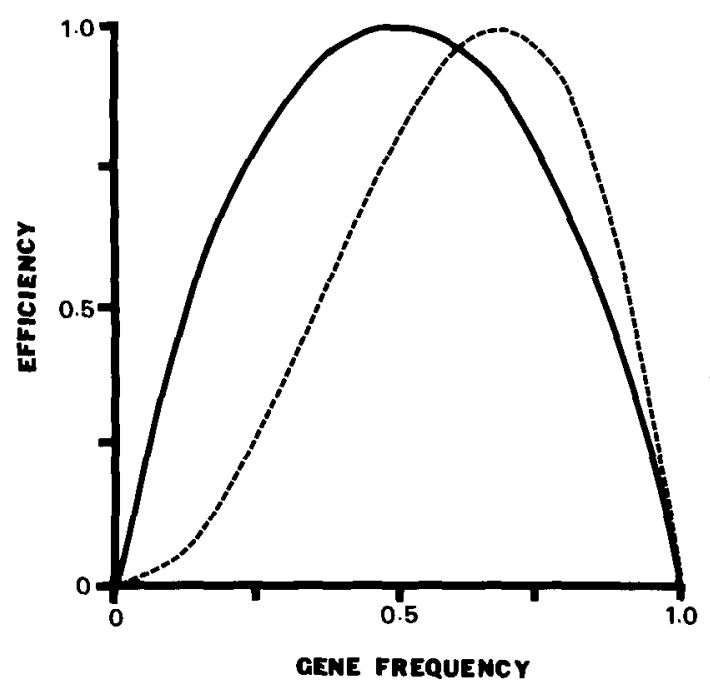

Figure. Selvin's measure of efficiency as a function of gene frequency for a diallelic autosomal locus. Solid (-): alleles codominant; broken line (- - ): one allele dominant over the other, gene frequency used is that for the recessive allele. 


\begin{tabular}{|c|c|c|c|c|c|c|c|c|c|}
\hline & 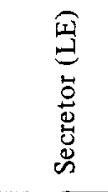 & 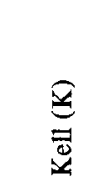 & $\begin{array}{l}\text { 齐 } \\
\text { 悹 } \\
\text { 音 }\end{array}$ & 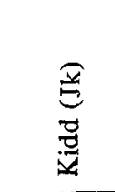 & a & $\stackrel{\circ}{\gtrless}$ & $\begin{array}{l}\circ \\
\dot{\infty} \\
\dot{Z}\end{array}$ & 总 & $\tilde{z}$ \\
\hline Num & 2 & 2 & 2 & 2 & 2 & 3 & 4 & 4 & 8 \\
\hline Recessiv & le & $\mathrm{k}$ & $\mathrm{Fy}^{\mathrm{b}}$ & - & $\mathrm{p}$ & 0 & 0 & - & $d, e$ \\
\hline Prob(concordance |DZ) & 0.7952 & 0.9047 & .7439 & 0.5938 & 0.7764 & 0.6615 & 0.6234 & 0.4406 & 0.4782 \\
\hline
\end{tabular}

values of these probabilities do not occur when all gene frequencies are equal (as Selvin noted), since at least in $\mathrm{ABO}$ and $\mathrm{Rh}$ loci not all genotypes are phenotypically distinguishable. For example, in the $\mathrm{ABO}$ locus, this probability is minimum when $\mathrm{A}, \mathrm{B}$, and $\mathrm{O}$ allele frequencies are approximately $0.31,0.31$, and 0.38 , respectively. For these allele frequencies the minimum value is 0.5608 , and not 0.4630 as Selvin reports. This error, apparently, emerged from the fact that he considered $\mathrm{A}, \mathrm{B}$, and $\mathrm{O}$ alleles as codominant ones, whereas the correct probability of concordance for a random DZ twin pair is to be computed by the formula as given in Neel and Schull [1] .

It may, therefore, be concluded that the probability of concordance given that the twin pair is DZ, by itself, should be a sufficient criterion to judge the usefulness of a genetic marker in the diagnosis of twin zygosity. The efficiency measure proposed by Selvin is a function not only of the number of alleles, but also of the nature of dominance relationships between the alleles at the locus and thus any comparison without the dominance taken into account can be quite misleading as is the case in Selvin's study.

Acknowledgments. Supported by US Public Health Service grants GM 19513 and CA 19311.

\section{REFERENCES}

1. Neel JV, Schull WJ (1974): "Human Heredity." Chicago: University of Chicago Press.

2. Race RR, Sanger R (1975): "Blood Groups in Man," 6th Ed. Oxford: Blackwell Scientific Publications.

3. Selvin S (1977): Efficiency of genetic systems for diagnosis of twin zygosity. Acta Genet Med Gemellol 26:81-82.

4. Smith SM, Penrose LS (1955): Monozygotic and dizygotic twin diagnosis. Ann Hum Genet 19: $273-289$.

Correspondence: Dr. R. Chakraborty, Center for Demographic and Population Genetics, University of Texas, PO Box 20334, Houston, TX 77025, USA. 